\title{
Estudio PIENSA: efecto de la asesoría sobre la elección de anticonceptivos hormonales combinados en mujeres chilenas
}

\author{
Ernesto Pizarro ${ }^{1}$, Guillermo Galán ${ }^{2}$, Pablo Lavín ${ }^{3}$, Claudio Benavides ${ }^{4}$, Francisca Rivera. ${ }^{5}$ \\ ${ }^{1}$ Ginecólogo Clínica Alemana de Santiago; ${ }^{2}$ Ginecólogo USACH; ${ }^{3}$ Ginecólogo Universidad de Chile; ${ }^{4}$ Ginecólogo \\ MSD Chile, USACH; ${ }^{5}$ Químico Farmacéutico MSD Chile.
}

DECLARACIÓN DE CONFLICTO DE INTERÉS: El estudio fue financiado por MSD. Los doctores Guillermo Galán y Ernesto Pizarro han sido speaker de laboratorios MSD, Bayer, Recalcine entre otros. El Dr. Pablo Lavín es asesor externo de MSD Chile. El Dr. Claudio Benavides y Francisca Rivera son empleados de MSD Chile.

\section{RESUMEN}

Objetivos: Evaluar la asesoría acerca de los anticonceptivos hormonales combinados de auto administración por parte de profesionales de la salud y cómo influye en el cambio o elección del método a utilizar. Método: Durante la consulta ginecológica habitual, se registra la elección del método anticonceptivo (MAC) combinado de auto administración (píldora, anillo o parche transdérmico) previa a la asesoría del médico y posterior a esta. El registro se realiza por medio de una encuesta que debe llenar la paciente y otra a completar por el médico. Resultados: Luego de la asesoría, de las 867 mujeres participantes un 11,6\% variaron su opción de MAC elegido previamente. La píldora, originalmente el método más elegido, disminuyó en las preferencias, aunque continuó siéndolo y las otras opciones aumentaron. La píldora anticonceptiva bajó en un $13,4 \%$ desde su elección inicial, el anillo vaginal y el parche transdérmico incrementaron su elección en un 63,6 y $56,7 \%$ respectivamente. Conclusión: Las píldoras son el MAC hormonal combinado preferido por las mujeres participantes. Sin embargo entregándo una información estandarizada se produce un impacto en la elección anticonceptiva, favoreciendo los métodos menos tradicionales y de uso prolongado como son el anillo vaginal y parche transdérmico.

\section{PALABRAS CLAVES: Anticoncepción, asesoría, píldora, parche, anillo vaginal}

\section{SUMMARY}

Objectives: To evaluate the impact of health care professional counseling in the choice of combined selfadministered contraceptive method. It also assesses how such advice influences in the final contraceptive choice or how it changes from the original. Method: During routine gynecological consultation, the patient's self-administered contraceptive method (pill, vaginal ring and transdermal patch) preference was registered. This was done before and after physician counseling, through a survey filled by both the patient and the physician. Results: After counseling, of the 867 women who participated, $11.6 \%$ changed their previously chosen contraceptive. The pill, originally the chosen method of the majority of women, decreased in terms of preference; although the pill continued to be the preferred contraceptive method, preference for other methods increased. The contraceptive pill decreased $13.4 \%$ in the preference rating, while the choice of the vaginal ring and the transdermal patch increased by 63.65 and $56.7 \%$ respectively. Conclusion: The pill is women's preferred combined self-administered contraceptive method. Nevertheless during counseling, a standardized information impacts in the contraceptive choice increasing the preference of less conventional methods as transdermal patch and vaginal ring.

\section{KEY WORDS: Contraception, counseling, pill, patch, vaginal ring}




\section{INTRODUCCIÓN}

En la elección e indicación de un método anticonceptivo (MAC) en la mujer se involucran dos pilares fundamentales. Estos son, por una parte, el equipo sanitario, el cual tiene el deber de ofrecer todos los métodos anticonceptivos disponibles, educando, aclarando y aconsejando respecto de las ventajas, desventajas y eventuales efectos secundarios de cada uno de los métodos ofrecidos, y por otro lado, las potenciales futuras usuarias, las cuales deberán hacer una elección de acuerdo a sus principios, creencias y deseos después de la información recibida. Todo lo anterior en el contexto de una evaluación clínica que permita que la elección definitiva no conlleve un riesgo en su uso y que los beneficios del método superen a los riesgos. Este concepto, denominado decisión conjunta informada, cada vez adquiere mayor relevancia a nivel mundial, y se involucra en los derechos que tienen los pacientes para ser correctamente asesorados en la elección de una pauta terapéutica, siendo en este caso específico un MAC.

Desafortunadamente, por problemas de recursos personales o por limitantes en los tiempos destinados a este fin por parte de los equipos sanitarios, esta situación no siempre puede ser cumplida, determinando que en algunos casos las mujeres puedan usar métodos anticonceptivos que podrían no ser los más adecuados para ellas y no satisfacer plenamente sus deseos y necesidades. Por otra parte, los embarazos no planificados son muy frecuentes en todo el mundo y las consecuencias como son el incremento en el número de abortos y patologías del embarazo las sufren las mujeres. Todo esto a pesar de la amplia variedad de métodos anticonceptivos disponibles y del incremento en el conocimiento acerca de ellos de parte de las usuarias, que se ha podido apreciar en los últimos años. Los estudios publicados muestran que parte importante de estos embarazos no planificados se producen por mal uso del MAC elegido o por discontinuidad de uso del mismo $(1,2)$. Es por todo esto que se diseña por parte de MSD (Merck Sharp \& Dohme), un programa y estudio multicéntrico a nivel latinoamericano con un protocolo común para ser desarrollado por 11 países latinoamericanos.

La píldora anticonceptiva hormonal combinada compuesta por un estrógeno más un progestágeno, es uno de los métodos más usados como anticonceptivo en países europeos y latinoamericanos (3). Su uso en el mercado mundial se remonta a los inicios de la década de los años sesenta. Más recientemente han aparecido en el mercado chileno anticonceptivos hormonales combinados en los que la clásica vía de administración oral ha sido innovada por la vía vaginal (anillo vaginal), o cutánea (parche transdérmico), los cuales tienen igualmente los elementos hormonales anteriormente mencionados $(4,5)$.

Chile, es un país de poco más de 17 millones de habitantes ubicado en el extremo suroccidente de Sudamérica. Las realidades de salud reproductiva y prevalencia de uso de métodos anticonceptivos, así como los tipos de anticonceptivos usados son bastante diversos entre los países latinoamericanos. Chile es un país con una vasta historia en uso de tecnología anticonceptiva que se materializa esencialmente desde 1965 en adelante, año en el que se establece un programa del Ministerio de Salud para la entrega gratuita de anticonceptivos al sector público de salud. Sin embargo, a pesar del programa de planificación familiar, siguen existiendo embarazos no deseados o no planificados, lo que constituye un grave problema de salud personal, familiar y social $(6,7)$.

La tasa de embarazos no deseados en adultos y adolescentes según un estudio latinoamericano fue de un $56 \%$ (8). Por otra parte, la elevada tasa de embarazos en las adolescentes no ha variado significativamente desde los años 1960 correspondiendo a un $15,6 \%$ (7), lo que representa uno de los problemas más serios de salud reproductiva y pública que debe enfrentar el país $(6,7)$. En Chile el aborto es ilegal en todas sus formas y está penalizado por ley, por lo que no hay estadísticas confiables sobre el tema.

En el sector público del país al año 2005, el $36 \%$ de las mujeres usaban anticonceptivos orales combinados, $54 \%$ dispositivo intrauterino, $7 \%$ otros métodos hormonales de su elección, y el $3 \%$ restante usaban métodos de barrera (6). A partir de ese año se empieza a producir un aumento progresivo en las prevalencias de uso de los anticonceptivos orales combinados, llegando a un $47,5 \%$ el año 2007 y al $54 \%$ en el 2009. En el sector público no se entrega gratuitamente ni el anillo vaginal ni el parche transdérmico al no estar considerado dentro de las políticas de salud pública. Es por esto que a pesar de que no hay cifras concretas, se estima que el uso de estos dos métodos anticonceptivos es mínimo.

En el sector privado de salud de Chile existe la posibilidad de adquirir toda una gama de métodos anticonceptivos como, píldoras combinadas con todos los progestágenos que puedan ser usados en ellas, píldoras que usan tanto estradiol como etinilestradiol, píldoras de progestágenos solos, inyectables mensuales combinados, inyectables de progestágeno solo que entregan una protección anticonceptiva de tres meses, implantes subcutáneos, dispositivos intrauterinos medicados con cobre o medicados con levonorgestrel, parche trasdérmico, anillo vaginal de solo progesterona o con 
la combinación de etinilestradiol más etonogestrel. La prevalencia de uso de cada uno de ellos en el sector privado es difícil de calcular ya que a pesar de que se debe comprar presentando una prescripción médica, en la práctica pueden ser adquiridos directamente en las farmacias locales. No obstante, se estima que la prevalencia de uso de la píldora anticonceptiva hormonal combinada es cercana a un $81 \%(9)$.

Este estudio se inspira en la pionera comunicación del estudio TEAM-06 realizado en 2007 por Lete en mujeres españolas (10), en que se pretenden iguales objetivos que los del estudio PIENSA. Le suceden a este estudio publicaciones similares, como el estudio IMAGINE en mujeres portuguesas, de Costa y cols (11) en 2011, y el estudio CHOICE de Bitzer y cols (12) en 11 países europeos. Este último estudio y otros realizados en esta década (13) han mostrado que a pesar de existir considerables diferencias entre los países participantes, después de haber recibido una adecuada orientación la elección anticonceptiva varía, especialmente la elección del anillo vaginal combinado y parche transdérmico aumentando respecto a aquellas mujeres que inicialmente consultaban solicitando la píldora anticonceptiva combinada, confirmando los hallazgos previos de Lete en España y de Costa en Portugal.

Los objetivos específicos del programa PIENSA son: A. Apoyar la asesoría acerca de los anticonceptivos hormonales combinados de auto administración por parte de profesionales de la salud. B. Evaluar los efectos de dicha asesoría en las decisiones de las mujeres respecto al método seleccionado. C. Cuantificar cuál método es elegido después de la información acerca de los métodos hormonales combinados de autoadministración disponibles (píldora, parche transdérmico, anillo vaginal). D. Comparar el método elegido con la intención original (método que la mujer pensó que elegiría antes de visitar a su médico). E. Describir los criterios usados por las mujeres para elegir su MAC. F. Valorar los indicadores sociales y demográficos de la elección anticonceptiva.

\section{SUJETOSY MÉTODOS}

El estudio PIENSA es un Programa de Investigación y Educación en Salud Anticonceptiva en mujeres que consultan por anticoncepción hormonal combinada de auto administración. En cada país participante, se formó un Comité Directivo Local. En el caso de Chile se contó con el apoyo de una Organización de Investigación por Contrato (CRO), la que fue responsable de la implementación del proyecto (distribución y recolección de cuestionarios completados por los profesionales y usuarias).

El grupo objeto de este estudio fueron mujeres con edades entre 18 y 40 años, quienes consultan a su ginecólogo por anticoncepción y considerarían un método hormonal anticonceptivo combinado de auto administración. Ellas consienten completar un cuestionario y que la información del cuestionario sea revisada y analizada por el personal que participa en el proyecto. En el caso de las mujeres que solicitaban un método anticonceptivo hormonal combinado, pero para las cuales el profesional de la salud consideró otro método más apropiado (debido a la presencia de contraindicaciones para anticoncepción hormonal combinada por ejemplo) (14), fueron asesoradas acerca de estos otros métodos pero aun así completaron el cuestionario.

El estudio se realizó en las oficinas o consultorios de práctica médica de los 38 profesionales gineco-obstetras participantes (sector oriente de Santiago). Los médicos fueron seleccionados aleatoriamente por el CRO a partir de una base de datos de 480 especialistas, y ellos fueron los que evaluaron y seleccionaron a las pacientes que participaron en el estudio. Además, tanto el protocolo como todos los materiales utilizados fueron presentados y aprobados por el comité de ética local.

El tamaño de muestra para el total de encuestas a realizar se determinó considerando la frecuencia del uso del anillo vaginal, nivel de confianza requerido $(90 \%)$, precisión del estudio $(2,5 \%)$, y considerando un $5 \%$ de no respuesta o datos perdidos. Estos datos se introdujeron en la ecuación utilizada para calcular la muestra en un muestreo aleatorio simple para un estudio descriptivo.

Durante el desarrollo del proyecto, los ginecólogos mantuvieron un registro de las mujeres que los consultaron por anticoncepción. Se solicitó la participación a las mujeres quienes considerarían un MAC combinado de auto administración (anillo, parche o píldora). Las mujeres que consultaron al ginecólogo para suspender el uso de alguno de los tres métodos hormonales combinados antes mencionados no fueron elegibles para participar, con excepción de aquellas usuarias de una píldora combinada quienes considerarían cambiar a otra píldora combinada. En la Figura 1 se muestra el flujograma del proceso.

Antes de recibir más información acerca de sus opciones anticonceptivas, el ginecólogo se aseguraba que la mujer entregara su consentimiento para participar en este programa, mediante la firma del consentimiento informado. El ginecólogo procedía entonces a preguntar a la mujer qué método elegía y registraba la respuesta en el cuestionario $A$ y a su vez se le solicitaba a la usuaria responder las preguntas 1 y 2 en el cuestionario $B$. Estas preguntas hacen referencia a que método es el que utilizará con mayor probabilidad, y grado de satisfacción de los métodos utilizados anteriormente. Todo el material utilizado en el proceso está disponible en caso de que quiera solicitarlo. 


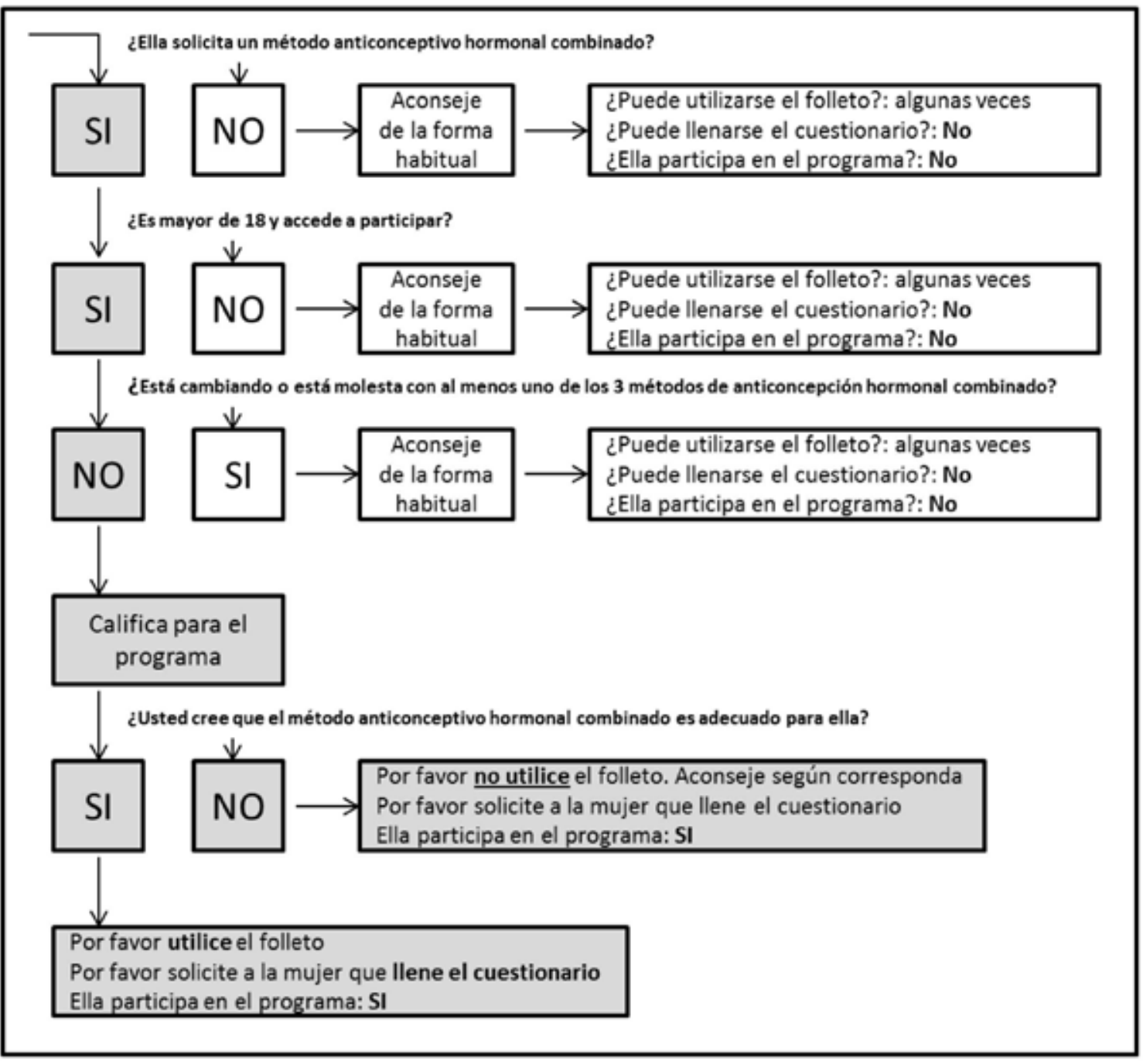

Figura 1. Flujograma del proceso de elección de mujeres participantes en el estudio.

El cuestionario $A$, busca que el médico registre la preferencia de MAC por parte de la usuaria antes de la asesoría, además de la existencia de alguna contraindicación de un MAC combinado y si se le entregó el folleto de asesoría a la usuaria. Por su parte, el cuestionario $B$ consta de dos partes, una que se debe llenar previa asesoría y otra posterior a esta. La primera parte tiene el objetivo de recabar la información demográfica de cada usuaria así como la percepción de ella sobre el anticonceptivo oral combinado, anillo vaginal y parche transdérmico. En el caso de la segunda parte se busca registrar las razones que tuvo la usuaria para elegir o no un método determinado.
Posteriormente y en la misma sesión, el ginecólogo asesoraba a la mujer acerca de los tres anticonceptivos hormonales combinados de auto administración disponible y tomaba nota en el cuestionario $A$ si es que había razones por las cuales los métodos combinados no eran adecuados, y sobre si se proporcionó a la mujer un folleto de asesoría. Excepto en los casos en que no fueran apropiados, el ginecólogo utilizaba los materiales de asesoría del programa para guiar la información verbal proporcionada. El material de asesoría y la discusión ofrecían una revisión justa y equilibrada de la efectividad de una variedad de métodos anticonceptivos, modo de acción, modos de uso, beneficios y riesgos potenciales 
e idoneidad con respecto a las necesidades individuales. Después de la discusión y la decisión sobre cuál método se prescribió, y siguiendo en la misma sesión, se solicitaba a la mujer completar el resto del "cuestionario B", incluyendo cuál método eligió finalmente, razones para seleccionar este método y percepciones acerca de los métodos anticonceptivos. Los cuestionarios completados se recolectaron $y$ analizaron en forma central.

\section{RESULTADOS}

De las 926 mujeres enroladas y que contestaron las encuestas, 60 fueron excluidas de los resultados finales pues no cumplían con los criterios de inclusión. El promedio de edad de las mujeres en este estudio fue $28,7 \pm 7,2$ años. La mayoría relata tener una pareja estable $(80,5 \%)$, tiene educación superior $(88 \%)$, con empleo remunerado $(70 \%)$ y el $71,5 \%$ respondió que la píldora anticonceptiva fue el último MAC utilizado (Tabla I).

La Tabla II muestra el cambio ocurrido entre el MAC elegido previamente a la asesoría y el escogido posteriormente a la información. Los cambios

Tabla I

\section{DATOS DEMOGRÁFICOS DE LAS PACIENTES PARTICIPANTES DEL ESTUDIO}

\begin{tabular}{lrr}
\hline Variables & $\mathrm{n}$ & $\%$ \\
\hline Pareja estable & & \\
$\mathrm{Si}$ & 697 & 80,5 \\
No & 169 & 19,5 \\
Nivel de educación & & \\
$\quad$ Primaria & 8 & 0,9 \\
$\quad$ Secundaria & 96 & 11,1 \\
$\quad$ Superior & 762 & 88,0 \\
Situación de empleo & & \\
$\quad$ Dueña de casa, estudia o sin & 262 & 30,3 \\
$\quad$ empleo & & \\
Medio Tiempo & 128 & 14,8 \\
$\quad$ Tiempo Completo & 476 & 55,0 \\
Último método anticonceptivo usado & & \\
Oral combinado & 619 & 71,5 \\
$\quad$ Anillo vaginal & 52 & 6,0 \\
$\quad$ Condón & 49 & 5,7 \\
Dispositivo intrauterino & 42 & 4,8 \\
Inyectable & 25 & 2,9 \\
Parche transdérmico & 21 & 2,4 \\
Implante subcutáneo & 7 & 0,8 \\
Ninguno & 51 & 5,9 \\
\hline
\end{tabular}

de la elección que ocurrieron en las mujeres luego de recibir la información se presentan en la Tabla III. La Tabla IV muestra el cambio de elección entre el último método usado y el que eligió la usuaria luego de la asesoría.

Dentro del cuestionario contestado por las usuarias, se les pedía que seleccionaran de un listado de aseveraciones, cuales correspondían a las principales razones para elegir o no elegir un método específico. En el caso de las razones esgrimidas para elegir el método en cuestión, se dijo: para la píldora anticonceptiva "sangrado menstrual regular" (58\%) y "acostumbrada a utilizarla" (56\%); para el anillo mensual "uso mensual" (73\%), "menos probabilidad de olvido" (66\%) y "fácil de usar" (65\%); mientras que para el parche transdérmico "fácil de usar" (64\%), "menos probabilidad de olvido" (62\%) y "uso semanal" $(47 \%)$.

Por otra parte, en cuanto a la no elección de un método la aseveración elegida con mayor frecuencia fue: para la píldora "olvido de uso" (75\%), para el anillo mensual "le incomoda la vía vaginal" (49\%), y en caso del parche transdérmico es considerado "poco discreto, visible" (43\%).

\section{DISCUSIÓN}

Este estudio es el primero que se realiza en Chile en que se evalúa el impacto que tiene la entrega de información estandarizada en la elección del MAC hormonal combinado de auto administración en la mujer. Tal como se comentó anteriormente, la píldora anticonceptiva es el MAC más utilizado en Chile con un $81 \%$, lo que hace sentido con los resultados obtenidos al ver que el mayor porcentaje lo obtuvo la píldora tanto para el último método usado $(71,4 \%)$, el método elegido previa a la asesoría $(75,5 \%)$ y el método elegido después de la asesoría $(65,5 \%)$.

Observamos un aumento en el número de mujeres que optaron por el anillo vaginal (de 12,3\% a $20,2 \%$ ) y el parche (de $3,5 \%$ a $5,4 \%$ ) y una reducción en las mujeres que optaron por el anticonceptivo oral combinado (de $75,5 \%$ a $65,5 \%$ ) entre la pre y la post asesoría (Tabla II). Esto describe el impacto que la asesoría genera en la elección del MAC.

Si bien la gran mayoría de las mujeres mantienen su elección inicial, el anillo vaginal combinado y el parche transdérmico fueron los métodos que se mantuvieron en mayor porcentaje $(92,5 \%$ y $93,3 \%$ respectivamente), mientras que la píldora anticonceptiva solo en un $84,1 \%$ (Tabla III). Esto indicaría que la mujer chilena dispone de información respecto al MAC que utiliza o desea utilizar. Respecto al último MAC utilizado, el anillo vaginal es el que presenta el mayor incremento porcentual después de la entrega de información, mientras que la píldora anticonceptiva presenta una disminución (Tabla IV). 


\section{Tabla II \\ MÉTODO ELEGIDO PRE Y POST ASESORÍA}

\begin{tabular}{|c|c|c|c|c|c|}
\hline \multirow[t]{2}{*}{ Método } & \multicolumn{2}{|c|}{$\begin{array}{c}\text { Elección previa a la } \\
\text { asesoría }\end{array}$} & \multicolumn{2}{|c|}{$\begin{array}{c}\text { Elección después de } \\
\text { asesoría }\end{array}$} & \multirow[t]{2}{*}{$\begin{array}{l}\text { Variación de elección } \\
\text { final sobre inicial (\%) }\end{array}$} \\
\hline & $\mathrm{n}$ & $\%$ & $\mathrm{n}$ & $\%$ & \\
\hline \multicolumn{6}{|l|}{ Anticonceptivo oral } \\
\hline combinado & 655 & 75,5 & 567 & 65,5 & $-13,4$ \\
\hline Anillo vaginal & 107 & 12,3 & 175 & 20,2 & 63,6 \\
\hline Parche & 30 & 3,5 & 47 & 5,4 & 56,7 \\
\hline Otros & 58 & 6,7 & 56 & 6,5 & $-3,4$ \\
\hline Ninguno / No sabe & 17 & 2,0 & 21 & 2,4 & 23,5 \\
\hline
\end{tabular}

\section{Tabla III \\ CAMBIO EN LA ELECCIÓN DESPUÉS DE LA ASESORÍA}

\begin{tabular}{lcccccccccccc}
\hline Método & \multicolumn{2}{c}{$\begin{array}{c}\text { Previo } \\
\text { asesoría }\end{array}$} & & $\begin{array}{c}\text { Anticonceptivo } \\
\text { oral } \\
\text { combinado }\end{array}$ & $\begin{array}{c}\text { Anillo } \\
\text { vaginal }\end{array}$ & Parche & & Otros & $\begin{array}{c}\text { Ninguno / } \\
\text { No sabe }\end{array}$ \\
& $\mathrm{n}$ & $\%$ & $\mathrm{n}$ & $\%$ & $\mathrm{n}$ & $\%$ & $\mathrm{n}$ & $\%$ & $\mathrm{n}$ & $\%$ & $\mathrm{n}$ & $\%$ \\
\hline Oral combinado & 655 & 100,0 & 551 & 84,1 & 66 & 10,1 & 17 & 2,6 & 10 & 1,5 & 11 & 1,7 \\
Anillo vaginal & 106 & 100,0 & 5 & 4,7 & 98 & 92,5 & 0 & 0,0 & 2 & 1,9 & 1 & 0,9 \\
Parche & 30 & 100,0 & 1 & 3,3 & 1 & 3,3 & 28 & 93,3 & 0 & 0,0 & 0 & 0,0 \\
Otros & 58 & 100,0 & 6 & 10,3 & 6 & 10,3 & 1 & 1,7 & 41 & 70,7 & 4 & 6,9 \\
Ninguno / No sabe & 17 & 100,0 & 4 & 23,5 & 4 & 23,5 & 1 & 5,9 & 3 & 17,6 & 5 & 29,4 \\
\hline
\end{tabular}

Tabla IV CAMBIO EN LA ELECCIÓN CON RESPECTO AL ÚLTIMO MÉTODO UTILIZADO

\begin{tabular}{|c|c|c|c|c|c|}
\hline \multirow[t]{2}{*}{ Método } & \multicolumn{2}{|c|}{ Último método usado } & \multicolumn{2}{|c|}{$\begin{array}{l}\text { Elección después } \\
\text { de asesoría }\end{array}$} & \multirow[t]{2}{*}{$\begin{array}{l}\text { Variación de elección final } \\
\text { sobre último método (\%) }\end{array}$} \\
\hline & $\mathrm{n}$ & $\%$ & $\mathrm{n}$ & $\%$ & \\
\hline \multicolumn{6}{|c|}{ Anticonceptivo oral } \\
\hline combinado & 619 & 71,4 & 567 & 65,5 & $-8,4$ \\
\hline Anillo vaginal & 52 & 6,0 & 175 & 20,2 & 236,5 \\
\hline Parche & 21 & 2,4 & 47 & 5,4 & 123,8 \\
\hline Otros & 123 & 14,2 & 56 & 6,5 & $-54,5$ \\
\hline Ninguno & 51 & 5,9 & 21 & 2,4 & $-58,8$ \\
\hline
\end{tabular}


Hay que destacar que el grupo de mujeres que llegó sin una decisión inicial de que MAC deseaba usar era minoritario y muy pequeño comparado con los grupos que elegían alguno de los otros métodos inicialmente. Esto hace que los resultados aquí obtenidos no sean comparables con aquellos de los estudios CHOICE, IMAGINE y TEAM-06. No obstante, en todos los estudios incluyendo el presentado, la elección del anillo anticonceptivo y parche transdérmico aumentan luego de la asesoría.

La población encuestada hace que este estudio tenga la limitante de estar enfocado en un sector específico de Santiago. Aquí se concentra gran parte del estrato socioeconómico medio alto, lo que hace que los resultados aquí obtenidos no reflejan necesariamente la realidad de la mayoría de las mujeres chilenas y no son representativos para el resto de la población del país.

Cabe mencionar que el listado inicial de médicos con el que se pobló la base de datos fue entregado por MSD y entre ellos la empresa externa seleccionó a los participantes. Dentro de los médicos contactados, hubo un alto número de ellos que no accedió a participar. Las principales razones para esto fueron porque no pudieron ser contactados directamente, por no disponer de tiempo suficiente para realizar el estudio, o porque creyeron no poder reunir el número de casos propuesto. La mayoría de los profesionales que participó recibió una pequeña cantidad de dinero en reconocimiento del mayor tiempo que debieron dedicar al proyecto. Queda pendiente responder la relación con la elección de Métodos Anticonceptivos Reversibles de Larga Duración (LARC por sus siglas en inglés).

\section{CONCLUSIÓN}

Los AOC son el MAC hormonal combinado preferido por las mujeres participantes. Sin embargo también se muestra que entregándole una información estandarizada a estas mujeres hay un impacto en la elección anticonceptiva, favoreciendo los métodos menos tradicionales y de uso prolongado como son el anillo vaginal y parche transdérmico. A pesar de la limitante antes enunciada, esta investigación evidencia la importancia de la asesoría médica al momento de la elección de un MAC, y que incentiva la elección conjunta del método entre la usuaria y el profesional de la salud.

RECONOCIMIENTOS: A los médicos y usuarias que participaron en el proyecto. Este proyecto cuenta con el apoyo científico de la FLASOG y con el apoyo financiero y organización de la compañía farmacéutica MSD.

\section{REFERENCIAS}

1. Rosenberg M, Waugh MS. Causes and consequences of oral contraceptive noncompliance. Am J ObstetGynecol 1999;180:276-9.
2. Santoyo Haro $S, 1$ Alvarado Saldivar G, Corral Ruíz JL, Pons Chirigliano JE, Córdova Egüez S, et al. Conocimientos generales y uso de métodos anticonceptivos en mujeres en edad reproductiva de 14 países en América Latina. Aten Fam 2007;14:12-6.

3. Rosenberg MJ, Waugh MS, Meehan TE. Use and misuse of oral contraceptives risk indicators: for poor pill taking and discontinuation. Contraception 1995;51:283-8.

4. Oddsson K, Leifels-Fischer B, de Melo NR, Wiel-Masson D, Bendetto C, Verhoeven CH, Dieben TO. Efficacy and safety of a contraceptive vaginal ring (NuvaRing) compared with a combined oral contraceptive: a 1-year randomized trial. Contraception 2005;71:17682.

5. Abrams LS, Skee D, Natarajan J, Wong FA. Pharmacokinetic overview of Ortho Evra ${ }^{\mathrm{TM}} / \mathrm{Evvr}^{\mathrm{TM}}$. Fertil Steril 2002;77:3-12.

6. Díaz S. Información sobre la situación de la Planificación Familiar en Chile. Chile. Instituto Chileno de Medicina Reproductiva (ICMER). (URL disponible el 16/Abr/2014 en: http://www.icmer.org/documentos/ anticoncepcion_de_emergencia/inf_situac_planific_ familiar_chile.pdf).

7. Subsecretaría de Salud Pública División de Prevención y Control de Enfermedades, Departamento de Ciclo Vital Programa Nacional de Salud Integral de Adolescentes y Jóvenes. Situación actual del embarazo adolescente en Chile. Chile: Ministerio de Salud (MINSAL); Julio 2013. (URL disponible el 16/Abr/14 en: http://www.mineduc.cl/usuarios/convivencia_escolar/doc/201210251259030.situacion_actual_embarazo_adolescente_en_chile.pdf).

8. The Alan Guttmacher Institute. Aborto clandestino: Una realidad latinoamericana. The Alan Guttmacher Institute. Nueva York; 1994.

9. Fuente: IMS Health; http://www.imshealth.com.

10. Lete I, Doval JL, Pérez-Campos E, Sánchez-Borrego $\mathrm{R}$, Correa $\mathrm{M}$, de la Viuda $\mathrm{E}$, et al. Factors affecting women's selection of a combined hormonal contraceptive method: The TEAM-06 Spanish cross-sectional study. Contraception 2007;76:77-83.

11. Costa AR, Palma F, Sá JL, Vicente L, Bombas T, Nogueira AM, Rocha P. Impact of a women's counselling programme on combined hormonal contraception in Portugal - the IMAGINE study. Eur J Contracept Reprod Health Care 2011;16:409-17.

12. BitzerJ, Gemzell-Danielsson K, Roumen F, Marintcheva-Petrova $M$, van Bakel $B$, Oddens BJ. The CHOICE study: effect of counselling on the selection of combined hormonal contraceptive methods in 11 countries. Eur J Contracept Reprod Health Care 2012;17:65-78.

13. Merckx M, Donders GG, Grandjean P, Van de Sande $\mathrm{T}$, Weyers $\mathrm{S}$. Does structured counselling influence combined hormonal contraceptive choice? Eur J Contracept Reprod Health Care 2011; 16:418-29

14. Organización Mundial de la Salud. Guías de Planificación Familiar para agentes de salud comunitarios y sus clientes. Ginebra: OMS; 2012. 